\title{
Tougu Xiaotong formula induces chondrogenic differentiation in association with transforming growth factor- $\beta 1$ and promotes proliferation in bone marrow stromal cells
}

\author{
JIASHOU CHEN ${ }^{1 *}$, GUOZHONG LIU ${ }^{2 *}$, XIAPING WENG ${ }^{1}$, FAYUAN LIU ${ }^{3}$, PINGDONG LIN $^{1}$, HUITING LI $^{1}$, \\ WENLIE CHEN ${ }^{3}$, YUNMEI HUANG ${ }^{4}$, XIANXIANG LIU ${ }^{3}$, HONGZHI YE $^{3}$ and XIHAI LI ${ }^{3}$ \\ ${ }^{1}$ College of Pharmacy, Fujian University of Traditional Chinese Medicine, Fuzhou, Fujian 350122; \\ ${ }^{2}$ The First Affiliated Hospital of Fujian Medical University, Fuzhou, Fujian 350005; \\ ${ }^{3}$ Academy of Integrative Medicine; ${ }^{4}$ Fujian Key Laboratory of Integrative Medicine on Geriatrics, \\ Fujian University of Traditional Chinese Medicine, Fuzhou, Fujian 350122, P.R. China
}

Received April 16, 2014; Accepted December 8, 2014

DOI: $10.3892 /$ ijmm.2014.2049

\begin{abstract}
Indian hedgehog (Ihh), one of the hedgehog gene families, is indicated in the regulation of chondrocyte differentiation. Tougu Xiaotong formula (TXF), a traditional Chinese medicinal compound, has been used for the treatment of bone and joint disease. However, the underlying molecular mechanisms of TXF on the function of bone marrow stromal cells (BMSCs) remain unclear. In the present study, the affect of TXF on proliferation and chondrogenic differentiation was investigated in primary BMSCs from four-week-old Sprague Dawley rats. The cell viability in BMSCs treated with TXF was higher compared to the untreated cells. Additionally, the percentage of $G_{0} / G_{1}$ phase cells was significantly decreased, whereas that of the $S$ phase cells was significantly increased. Furthermore, following TXF treatment, cyclin D1, cyclin-dependent kinase 4 (CDK4) and CDK6 expression in BMSCs was significantly enhanced. The results showed that TXF had no cytotoxicity to BMSCs. To explore the effect of TXF on the differentiation in BMSCs, whether TXF induced chondrogenic differentiation of BMSCs by the regulation of Ihh signaling pathway was investigated. The protein expression of Ihh, Patched and Smoothened in the induction group were significantly increased when compared to those in the
\end{abstract}

Correspondence to: Professor Hongzhi Ye or Dr Xihai Li, Academy of Integrative Medicine, Fujian University of Traditional Chinese Medicine, 1 Huatuo Road, University Town, Shangjie Minhou, Fuzhou, Fujian 350122, P.R. China

E-mail: yelin0930@163.com

E-mail: lixihai79dahai@163.com

${ }^{*}$ Contributed equally

Key words: Tougu Xiaotong formula, bone marrow stromal cells, chondrogenic differentiation, Indian hedgehog control group, and the highest protein level of Ihh was in the induction group that was treated with the combination of TXF and transforming growth factor- $\beta 1$ (TGF- $\beta 1$ ). In addition, TXF combined with TGF- $\beta 1$ significantly induced the protein expression of cartilage oligomeric matrix protein and collagen II compared to the TGF- $\beta 1$ group. Taken together, these results indicate that TXF promotes the proliferation via accelerating the $G_{1} / S$ transition, and induces chondrogenic differentiation in BMSCs by activation of the Ihh signaling pathway in association with TGF- $\beta 1$.

\section{Introduction}

Bone marrow stromal cells (BMSCs), a population of cells that have the capability of self-renewal and plasticity in vitro, can differentiate into multiple cell lineages in specific conditions, such as osteocytes, chondrocytes, fibroblasts and adipocytes (1-3). BMSCs are characterized by positive expression of surface markers, including cluster of differentiation 105 (CD105), CD44, CD73, CD90, CD29 and CD56, and negative expression of hematopoietic stem cell surface markers, such as CD45, CD34 and CD14 (4). Recently, BMSCs have attracted significant attention as an alternative to autologous chondrocytes for the repair of articular cartilage within the field of cartilage tissue engineering $(5,6)$. For successful chondrogenic differentiation of BMSCs, the cells should be induced by various factors, and among these Chinese herbs are a potential. Previous studies reported that an effective component of Chinese herbs was an inductive agent for chondrogenic differentiation of mesenchymal stem cells (7-9).

In particular, Indian hedgehog (Ihh), a member of the vertebrate hedgehog gene family to control the cartilage hypertrophy (10), plays a key role in the regulation of the chondrocyte proliferation and differentiation during endochondral bone formation $(11,12)$. Ihh is mainly produced by pre-hypertrophic and hypertrophic chondrocytes and binds to membrane receptor Patched (Ptc), which acts sub-stoichiometrically to suppress Smoothened (Smo) activity in the absence of Ihh signal. Upon Ihh binding to Ptc, Smo is activated to transmit 
signals downstream, which results in transcription regulation of the target genes of the hedgehog signaling pathway (13-15).

Tougu Xiaotong formula (TXF), a hospital preparation of the Second People's Hospital of Fujian Province (Fuzhou, China) consisting of 4 component herbs, including Morindae officinalis, Radix paeoniae alba, Ligusticum wallichii and Herba sarcandrae glabrae, has been proven to ameliorate the progress of cartilage degeneration by the regulation of chondrocyte autophagy and apoptosis $(16,17)$. However, the exact molecular mechanisms of the therapeutic effects of TXF remain unclear. Since the cell cycle plays an important role in the proliferation of BMSCs $(18,19)$, the present study results showed that TXF promoted BMSC proliferation by inducing the $G_{1} / S$ transition. TXF was also found to mediate BMSCs to chondrogenic differentiation by activating the Ihh signaling pathway.

\section{Materials and methods}

Animals. Four-week-old male Sprague Dawley (SD) rats were purchased from the Shanghai Slack Laboratory Animal Co. (Shanghai, China); animal permit number: SCXK (Shanghai, China) 2007-0005. All the experiments involving the animals complied with the Guidance Suggestions for the Care and Use of Laboratory Animals (2006) administered by the Ministry of Science and Technology of the People's Republic of China.

Herbal preparation. All the herbs of TXF were purchased from the Third People's Hospital of Fujian Province (Fuzhou, China). First, the original herbs were dried for $24 \mathrm{~h}$ in an air-circulating oven (model SFG-02.600; Hengfeng, Huangshi, China) at $50^{\circ} \mathrm{C}$. They were subsequently shredded further and then crushed to the appropriate particle size in a high-speed rotary cutting mill (model ZN-400A; Zhongnan, Changsha, China). According to the proportion of the TXF formula (2:2:1:1), $108 \mathrm{~g}$ of herbal powder was extracted with $1,500 \mathrm{ml}$ distilled water by refluxing twice, for $2 \mathrm{~h}$ each time. The undissolved materials were removed by filtration with Whatman filter paper, and the filtrate was evaporated on a rotary evaporator (model RE-2000; Yarong, Shanghai, China). The concentrated solution was dried to a constant weight in a vacuum drying oven (model DZF-300; Yiheng, Shanghai, China) after the filtrate was concentrated to a relative density of 1.25. The mother liquor of the TXF extracts was prepared by dissolving the extract powder in Dulbecco's modified Eagle's medium (DMEM; HyClone, Carlsbad, CA, USA) to a concentration of $20 \mathrm{mg} / \mathrm{ml}$.

High-performance liquid chromatography (HPLC) analysis. The study established a method of fingerprint analysis for quality control of TXF through detecting the HPLC fingerprint of the extracts of 10 TXF herb batches by an Agilent 1200 HPLC system (Agilent Technologies, Santa Clara, CA, USA), and the common peak was analyzed by the 'Similarity Evaluation System for Chromatographic Fingerprint of TCM' software (version 2004A). HPLC was performed using an Ultimate $^{\mathrm{TM}}$ XB-C18 column $(250 \times 4.6 \mathrm{~mm}, 5 \mu \mathrm{m})$. Methanol (solvent A) and $0.1 \%$ phosphoric acid (solvent $\mathrm{B}$ ) were the mobile phase and the detection wavelength was $277 \mathrm{~nm}$, the flow rate was $1 \mathrm{ml} / \mathrm{min}$ and the column temperature was $30^{\circ} \mathrm{C}$. The gradient procedure was as follows: $5 \% \mathrm{~A}$ at $0-5 \mathrm{~min}, 5-20 \% \mathrm{~A}$ at 5-10 $\mathrm{min}, 20-42 \% \mathrm{~A}$ at $15-25 \mathrm{~min}, 42-65 \% \mathrm{~A}$ at $25-40 \mathrm{~min}$, $65-80 \% \mathrm{~A}$ at $40-55 \mathrm{~min}$ and $80-100 \% \mathrm{~A}$ at $55-70 \mathrm{~min}$.

Isolation, culture and identification of rat BMSCs. Extraction and separation between BMSCs was performed using a complete marrow direct culture method. The SD rats were sacrificed by breaking the neck. The femurs and tibias were separated under sterile conditions and immersed in $75 \%$ alcohol for $20 \mathrm{~min}$. Following the separation of the muscles and tendons from the femurs and tibias, the marrow cavity was rinsed repeatedly with DMEM into $15 \mathrm{ml}$ centrifuge tubes and centrifuged at $160 \mathrm{x}$ g for $5 \mathrm{~min}$ to obtain a cell pellet. The supernatant fluid was removed and the cells were resuspended in DMEM with $15 \%$ fetal bovine serum, $100 \mathrm{U} / \mathrm{ml}$ penicillin and $100 \mu \mathrm{g} / \mathrm{ml}$ streptomycin (all from HyClone). The primary cells were seeded in culture flasks and cultured at $37^{\circ} \mathrm{C}$ in a $5 \% \mathrm{CO}_{2}$ incubator (termed P0). The cells were observed under a phase-contrast microscope (Olympus, Tokyo, Japan) and subcultured when they reached $80 \%$ confluency after 8 days (termed P1). The 3rd generation of BMSCs was identified using flow cytometry (Becton-Dickinson, San Jose, CA, USA), which measured the expression of BMSC surface marker, CD90, and hematopoietic stem cell surface marker, CD45.

Experimental design. The 3rd generation of BMSCs were collected and stimulated with various concentrations of TXF $(0-1,600 \mu \mathrm{g} / \mathrm{ml})$ for 24,48 and $72 \mathrm{~h}$, and the changes of the cell cycle were analyzed.

For chondrogenic differentiation, the cells were incubated in DMEM with $50 \mu \mathrm{g} / \mathrm{ml}$ vitamin $\mathrm{C}$ and $10^{-7} \mathrm{~mol} / \mathrm{ml}$ dexamethasone (Sigma, St. Louis, MO, USA) [auxiliary-induced medium (AIM)]. BMSCs were divided into 6 groups; control, $200 \mu \mathrm{g} / \mathrm{ml}$ TXF, $200 \mu \mathrm{g} / \mathrm{ml} \mathrm{TXF}+\mathrm{AIM}, 10 \mathrm{ng} / \mathrm{ml}$ transforming growth factor- $\beta 1$ (TGF- $\beta 1$ ) (PeproTech, Rocky Hill, $\mathrm{NJ}, \mathrm{USA})+\mathrm{AIM}, 10 \mathrm{ng} / \mathrm{ml}$ TGF- $\beta 1+200 \mu \mathrm{g} / \mathrm{ml}$ TXF + AIM and AIM groups. All the groups were treated once every 2 days for 2 weeks.

Cell viability analysis. Following treatment with TXF, $100 \mu \mathrm{l}$ 3-(4,5-dimethylthiazol-2-yl)-2,5-diphenyltetrazolium bromide (MTT) $[0.1 \mathrm{mg} / \mathrm{ml}$ in phosphate-buffered saline (PBS)] was added to each well, and the samples were incubated at $37^{\circ} \mathrm{C}$ for $4 \mathrm{~h}$. The purple-blue MTT formazan precipitate was dissolved in $150 \mu \mathrm{l}$ dimethyl sulfoxide and the 96-well plate was agitated for $10 \mathrm{~min}$. The absorbance was measured at $490 \mathrm{~nm}$ using an enzyme-linked immunosorbent assay reader (model EXL800; BioTek, Winooski, VT, USA).

Cell cycle analysis. The cell cycle of BMSCs was determined by flow cytometric analysis using a fluorescence-activated cell sorting (FACS) caliber. Propidium iodide (PI) staining was performed according to the manufacturer's instructions for the cell assay kit (KeyGen, Nanjing, China). The percentage of cells in the different phases was calculated by the ModFit software (Verity Software House, Topsham, ME, USA), and the cell numbers in the $G_{0} / G_{1}, S$ and $G_{2} / M$ phases were obtained.

Reverse transcription-polymerase chain reaction ( $R T-P C R)$ analysis. Total RNA was isolated with TRIzol reagent 

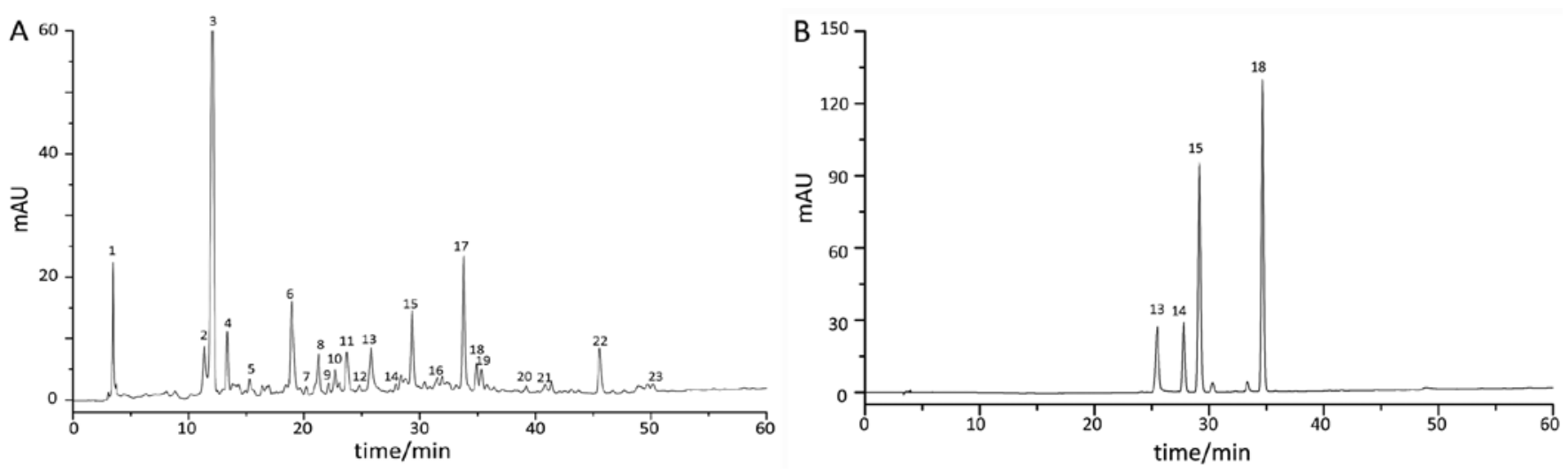

Figure 1. Fingerprint chromatography of (A) Tougu Xiaotong formula (TXF) and (B) the reference substance.

(Thermo Fisher Scientific, Waltham, MA, USA). RNA (2 $\mu \mathrm{g})$ was reverse transcribed into cDNA using a First Strand cDNA Synthesis kit (Thermo Fisher Scientific). The obtained cDNA was used to determine the mRNA levels of cyclin D1, cyclin-dependent kinase $4(C D K 4)$ and $C D K 6$. $\beta$-actin was used as an internal control. The sequences of the primers used for amplification of cyclin D1, CDK4, CDK6 and $\beta$-actin (Sangon Biotech, Shanghai, China) were as follows: Cyclin D1 forward, 5'-AAT GCC AGA GGC GGA TGA GA-3' and reverse, 5'-GCT TGT GCG GTA GCA GGA GA-3', 189 base pairs (bp); $C D K 4$ forward, 5'-GAA GAC GAC TGG CCT CGA GA-3' and reverse, 5'-ACT GCG CTC CAG ATT CCT CC-3', 109 bp; CDK6 forward, 5'-TTG TGA CAG ACA TCG ACG AG-3' and reverse, 5'-GAC AGG TGA GAA TGC AGG TT-3', 151 bp; and $\beta$-actin forward, 5'-GAG AGG GAA ATC GTG CGT GAC-3' and reverse, 5'-CAT CTG CTG GAA GGT GGA CA-3', 453 bp.

Immunohistochemistry (IHC) analysis. Chondrogenic differentiation of BMSCs was induced on the coverslips (Cosmobrand, Beijing, China) after 2 weeks. IHC was applied to identify that BMSCs differentiated to chondrocytes by detecting the collagen II expression. IHC was performed according to the manufacturer's instructions for the Polink-2 plus ${ }^{\circledR}$ polymer horseradish peroxidase (HRP) detection system (GBI Labs, Mukilteo, WA, USA). The cells on the coverslips were fixed for $30 \mathrm{~min}$ by $4 \%$ paraformaldehyde $\left(4^{\circ} \mathrm{C}\right)$, treated in $3 \% \mathrm{H}_{2} \mathrm{O}_{2}$ for 10 min (room temperature), blocked with $10 \%$ sheep serum for $30 \mathrm{~min}$ (room temperature) and incubated in primary antibody solution collagen II (BS1071; Bioworld Technology, St. Louis Park, MN, USA) overnight $\left(4^{\circ} \mathrm{C}\right)$. The cells on the coverslips were treated with Polymer Helper, poly-HRP anti-rabbit immunoglobulin $\mathrm{G}$ and 3,3'-diaminobenzidine for 20, 30 and $10 \mathrm{~min}$, respectively. Finally, Hematoxylin (Sigma) was redyed in each coverslips. To exclude any non-specific staining, PBS was used to replace the collagen II as the negative control. Images were captured using a phase-contrast microscope (magnification, x100; Olympus).

Western blot analysis. The cells were suspended in western blotting lysis buffer for $30 \mathrm{~min}$. After centrifugation at $20,000 \times \mathrm{g}$ at $4^{\circ} \mathrm{C}$ for $15 \mathrm{~min}$, the supernatant was collected. The protein concentration was determined by the bicinchoninic acid protein assay (Beyotime, Shanghai, China). Equal amounts of protein $(20 \mu \mathrm{g})$ were separated by electrophoresis on $12 \%$ SDS-polyacrylamide gels and transferred onto PVDF membranes (Millipore, Billerica, MA, USA). The membranes were blocked with blocking solution (5\% skimmed milk powder) for $2 \mathrm{~h}$ at room temperature and incubated in primary antibody solution of cyclin D1 (BS2436; Bioworld Technology), CDK4 (sc-260; Santa Cruz Biotechnology, Inc., Santa Cruz, CA, USA), CDK6 (14052-1-AP; Proteintech Group, Inc., Chicago, IL, USA), Ihh (sc-1196) and Ptc (sc-6149; both from Santa Cruz Biotechnology), cartilage oligomeric matrix protein (COMP) (5641-1; Epitomics, Burlingame, CA, USA) Smo (BS3428; Bioworld Technology), collagen II and $\beta$-actin (AP0060; Bioworld Technology) overnight at $4^{\circ} \mathrm{C}$, and subsequently with appropriate HRP-conjugated secondary antibody followed by enhanced chemiluminescence detection. Finally, protein images were captured and analyzed by a motored molecular imaging system (model GEL DOC 2000; Bio-Rad, Hercules, CA, USA).

Statistical analysis. SPSS 13.0 (SPSS, Inc., Chicago, IL, USA) was used for all the statistical analysis. All the data represented at least three independent experiments and statistical analysis of the data was performed with analysis of variance. Differences with $\mathrm{P}<0.05$ were considered to indicate a statistically significant difference.

\section{Results}

Fingerprint chromatography of TXF. The samples and reference substance were diluted in methanol and were determined by an Agilent 1200 HPLC system. Results found that 23 common peaks were analyzed in the fingerprint chromatography of 10 TXF batches using the 'Similarity Evaluation System for Chromatographic Fingerprint of TCM' software (version 2004A), and 4 compositions were identified from common peaks by comparing the retention time of the chromatographic peak between the sample and reference substance (Fig. 1). The reference substance was composed of paeoniflorin (peak 13), isofraxidin (peak 14), ferulic acid (peak 15) and rosmarinic acid (peak 18).

Morphological observation and identification of BMSCs. BMSCs were easily isolated from bone marrow and expanded in culture medium by the whole bone marrow adherent culture method $(20,21)$. The P0 cells had small amounts of adherence 

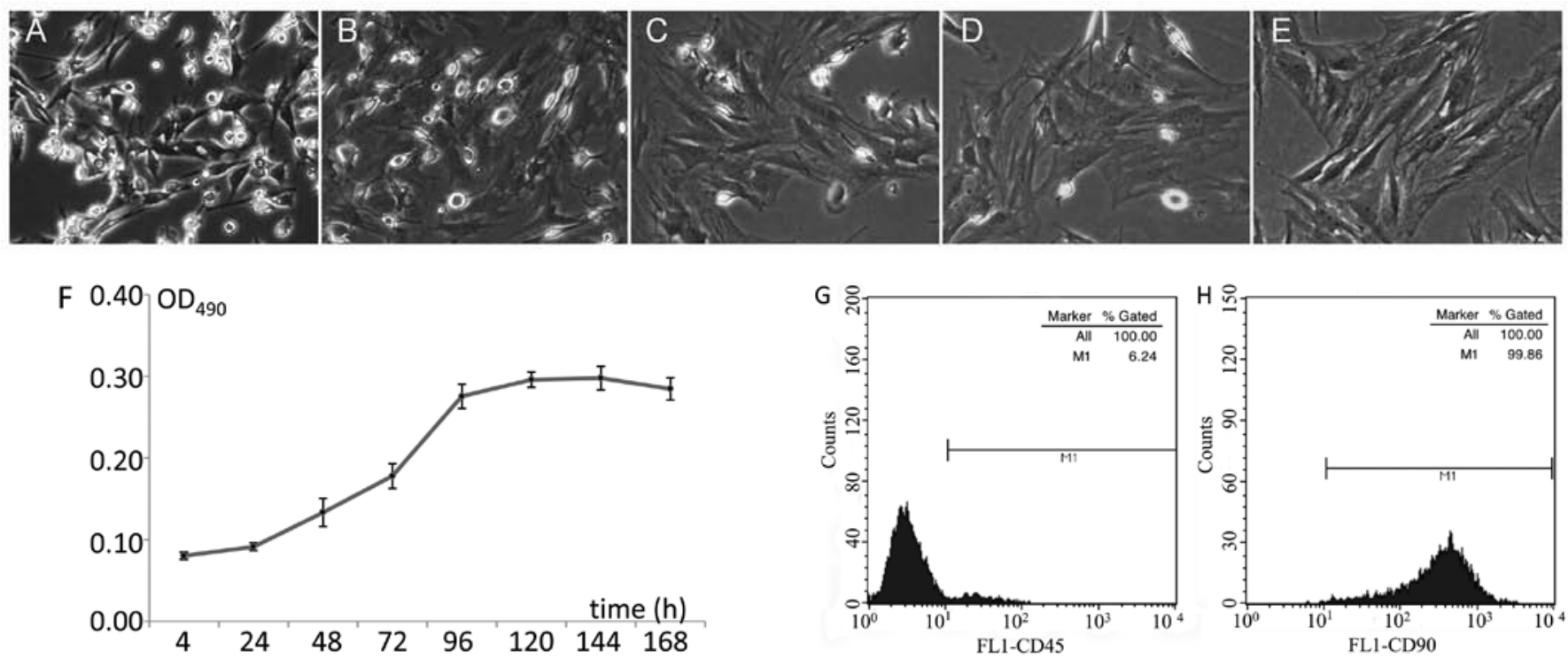

Figure 2. Morphological observation (magnification, $\mathrm{x} 100$ ) and identification of bone marrow stromal cells (BMSCs). The P0 BMSCs cultured for (A) 3 and (B) 8 days (P0). The (C) P1, (D) P2 and (E) P3 BMSCs cultured for 2 days. (F) The growth curve of BMSCs (P3). (G) Cluster of differentiation 45 (CD45) and (H) CD90 expression.
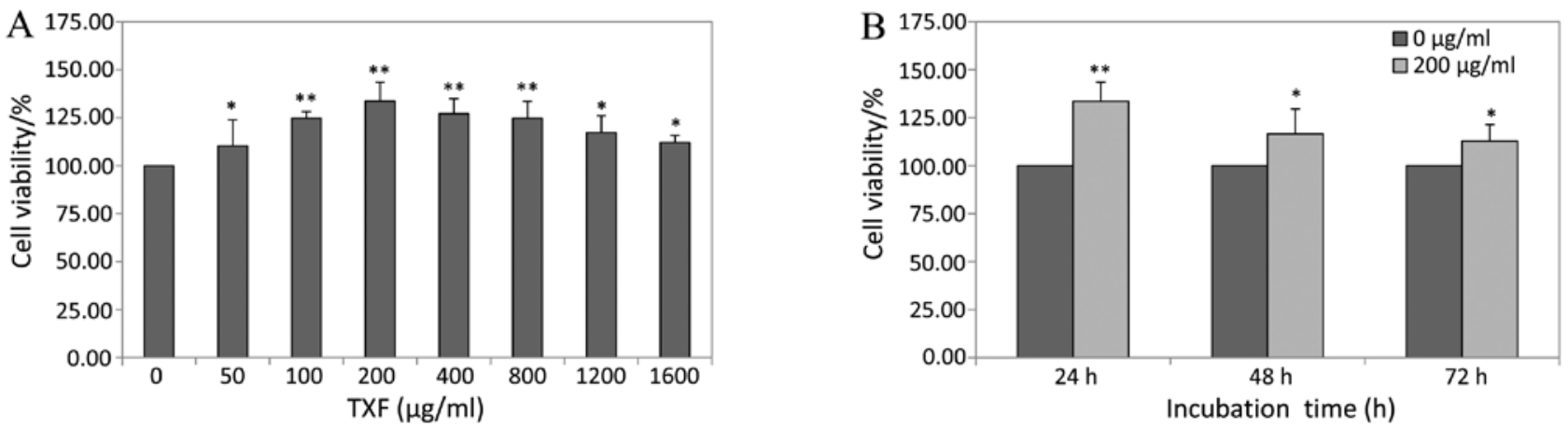

Figure 3. Tougu Xiaotong formula (TXF) enhances the bone marrow stromal cell (BMSC) viability. (A) BMSCs were treated with various concentrations of TXF for $24 \mathrm{~h}$. (B) BMSCs were treated with $200 \mu \mathrm{g} / \mathrm{ml}$ TXF for 24, 48 and $72 \mathrm{~h}$. Data represent mean \pm standard deviation of at least triplicate experiments. ${ }^{*} \mathrm{P}<0.05,{ }^{* *} \mathrm{P}<0.01$, vs. control.

after day 1 and formed round or polygon shapes, and subsequently had a large number of adherent cells accompanied by short spindle cells on day 3 (Fig. 2A). The cells were subcultured when they reached $80 \%$ confluence after 8 days (Fig. 2B). The size and shape of the P0 cells were different, however, becoming increasingly more uniform with increasing passages (Fig. 2C-E). CD90 and CD45 expression was detected for the identification of BMSCs by the flow cytometry assay. The P3 cells exhibited a positive expression of the BMSC surface marker, CD90, and negative expression of the hematopoietic stem cell surface marker, CD45 (Fig. 2G and H), and demonstrated significant reproductive activity (Fig. 2F). Therefore, the third generation of BMSCs were used in the subsequent experiments.

TXF enhances BMSC viability. The effect of TXF on the viability of BMSCs was determined by the MTT assay. Treatment with 50-1,600 $\mu \mathrm{g} / \mathrm{ml}$ TXF for $24 \mathrm{~h}$ increased cell viability by $10-34 \%$, and treatment with $200 \mu \mathrm{g} / \mathrm{ml} \mathrm{TXF}$ for 24 , 48 and $72 \mathrm{~h}$ increased cell viability by $33.59 \pm 2.47,14.31 \pm 1.53$ and $12.74 \pm 1.03 \%$, respectively $(\mathrm{P}<0.01$ or $\mathrm{P}<0.05$ vs. untreated cells), suggesting that TXF effects the viability in a dose- and time-dependent manner (Fig. 3).

TXF promotes the $G_{I} / S$ transition of BMSCs. $\mathrm{G}_{1} / \mathrm{S}$ transition is one of the two main checkpoints that regulate the progression of the cell cycle (22). To determine the mechanism of the proliferative activity of TXF, its effect on the $\mathrm{G}_{1}$ to $\mathrm{S}$ phase transition in BMSCs was analyzed via PI staining followed by FACS analysis. After simulation for $24 \mathrm{~h}$, the percentage of $\mathrm{G}_{0} / \mathrm{G}_{1}$ phase cells treated with 50, 100 and $200 \mu \mathrm{g} / \mathrm{ml}$ TXF $(52.82 \pm 2.56$, $34.27 \pm 3.12$ and $30.16 \pm 2.93 \%$ ) was significantly lower than that of the untreated control cells $(70.68 \pm 3.97 \%$; $\mathrm{P}<0.01)$, while the $S$ phase cells in the treated cells were higher than that of the untreated cells (Fig. 4). The results showed that TXF induced the proliferation of BMSCs by stimulating the $\mathrm{G}_{1} / \mathrm{S}$ transition.

TXF promotes cyclin D1, CDK4 and CDK6 expression. Cyclin D1, CDK4 and CDK6 are key regulators of the $\mathrm{G}_{1} / \mathrm{S}$ transition (23). The mRNA and protein expression of cyclin D1, CDK4 and CDK6 were analyzed by RT-PCR and western blotting, and the cyclin D1, CDK4 and CDK6 mRNA 

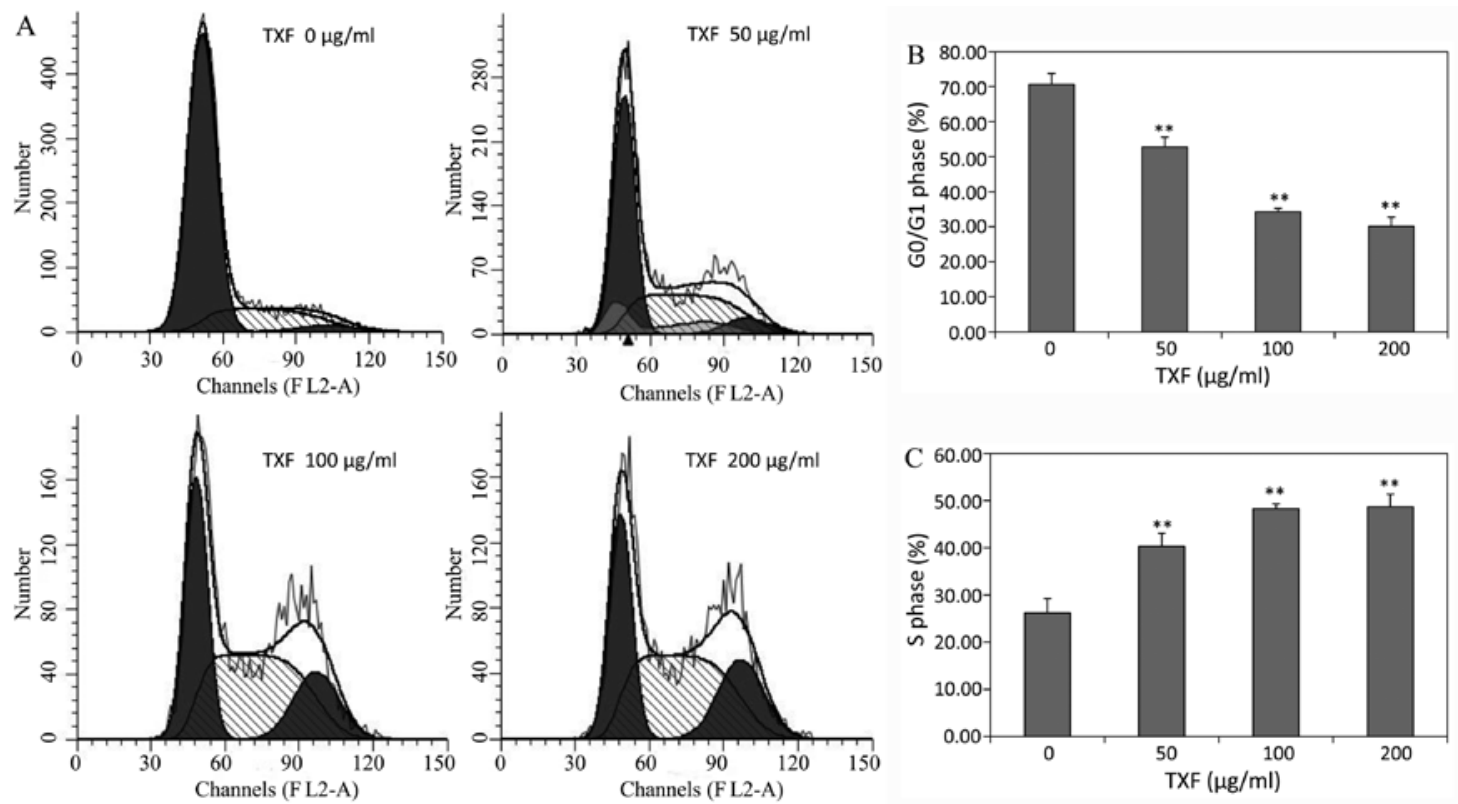

Figure 4. Tougu Xiaotong formula (TXF) promotes $\mathrm{G}_{1} / \mathrm{S}$ transition of bone marrow stromal cells (BMSCs). (A) BMSCs were collected and stained with propidium iodide (PI) staining followed by fluorescence-activated cell sorting (FACS) analysis. (B) The percentage of BMSCs in the $\mathrm{G}_{0} / \mathrm{G}_{1}$ phase cells following treatment with TXF. (C) The percentage of BMSCs in the S phase cells following treatment with TXF. The data shown are mean \pm standard deviation; ${ }^{*} \mathrm{P}<0.05,{ }^{* *} \mathrm{P}<0.01$, vs. control.
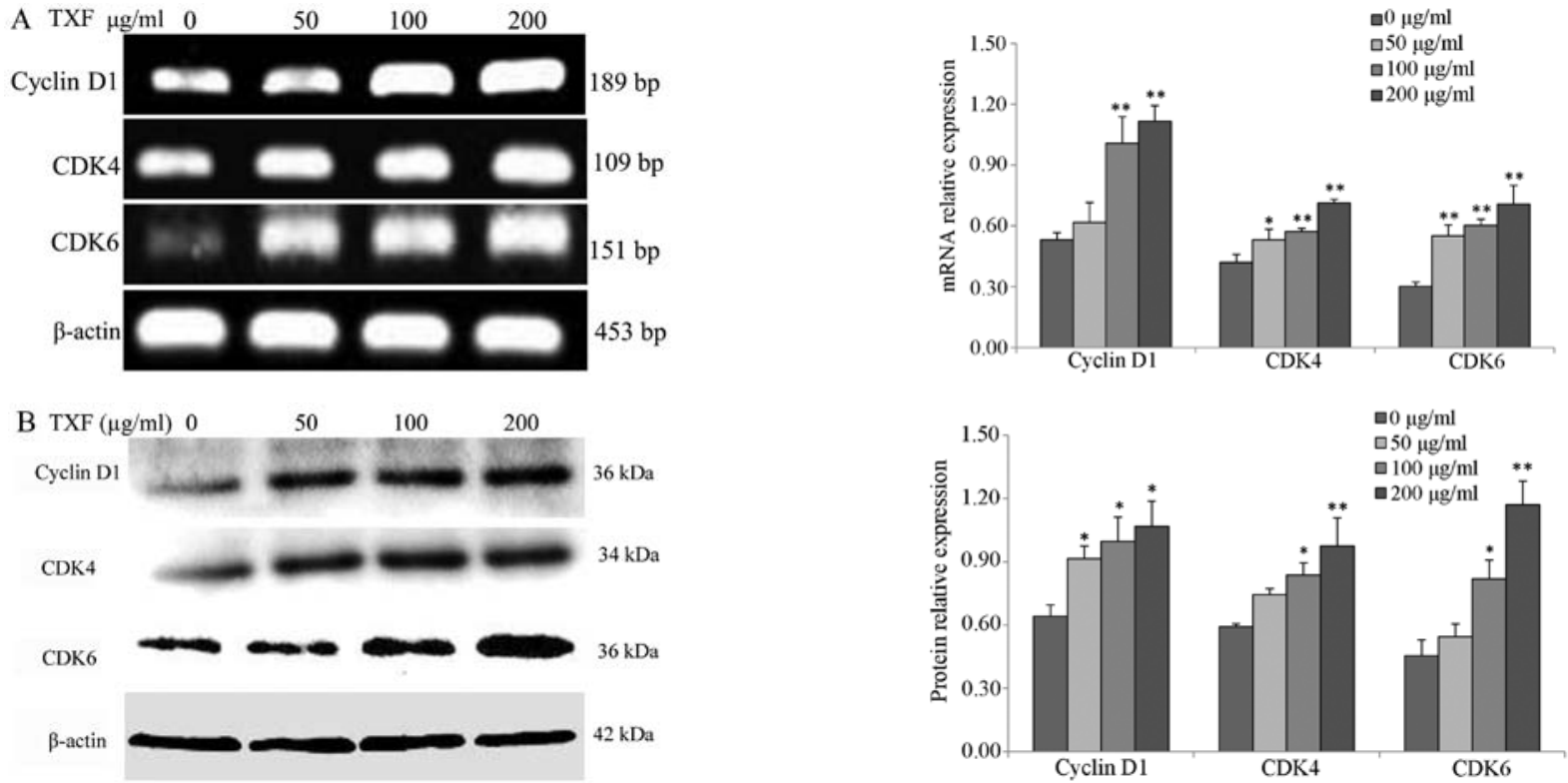

Figure 5. Tougu Xiaotong formula (TXF) upregulates cyclin D1, CDK4 and CDK6 expression in bone marrow stromal cells (BMSCs). (A) mRNA expression of cyclin D1, CDK4 and CDK6 was analyzed by RT-PCR, and $\beta$-actin was used as the internal control for the RT-PCR assays. (B) The protein expression levels of cyclin D1, CDK4 and CDK6 were determined by western blotting, and $\beta$-actin was used as the internal control for the western blot assays. The data shown are mean \pm standard deviation; ${ }^{*} \mathrm{P}<0.05,{ }^{* *} \mathrm{P}<0.01$, vs. control.CDK, cyclin-dependent kinase; RT-PCR, reverse transcription-polymerase chain reaction.

expression in BMSCs treated with TXF was higher compared to the untreated cells $(\mathrm{P}<0.01$ or $\mathrm{P}<0.05)$ (Fig. $5 \mathrm{~A})$; and the protein expression patterns of cyclin D1, CDK4 and CDK6 were similar to their respective mRNA expression, respectively (Fig. 5B). The results indicated that TXF promoted BMSCs from the $\mathrm{G}_{1}$ to the $S$ phase by upregulating the expression of cyclin D1, CDK4 and CDK6.

Immunohistochemistry detection of chondroblast-like cells. Collagen II is produced by chondrocytes (24), and increased collagen II expression is an indicator for differentiation of BMSCs into chondrocytes. Collagen II in the extracellular matrix (ECM) was determined by IHC. There were no collagen II positive cells in the basal medium (control) (Fig. 6). Following culture in AIM, BMSCs began to synthesize a small amount of collagen II after 2 weeks, and after TXF, TGF- $\beta 1$ or TGF- $\beta 1+$ TXF were added to AIM, collagen II positive cells clearly increased. Conversely, only a small increase of collagen II positive cells was found following the independent application of TXF. The TXF + AIM group increased 


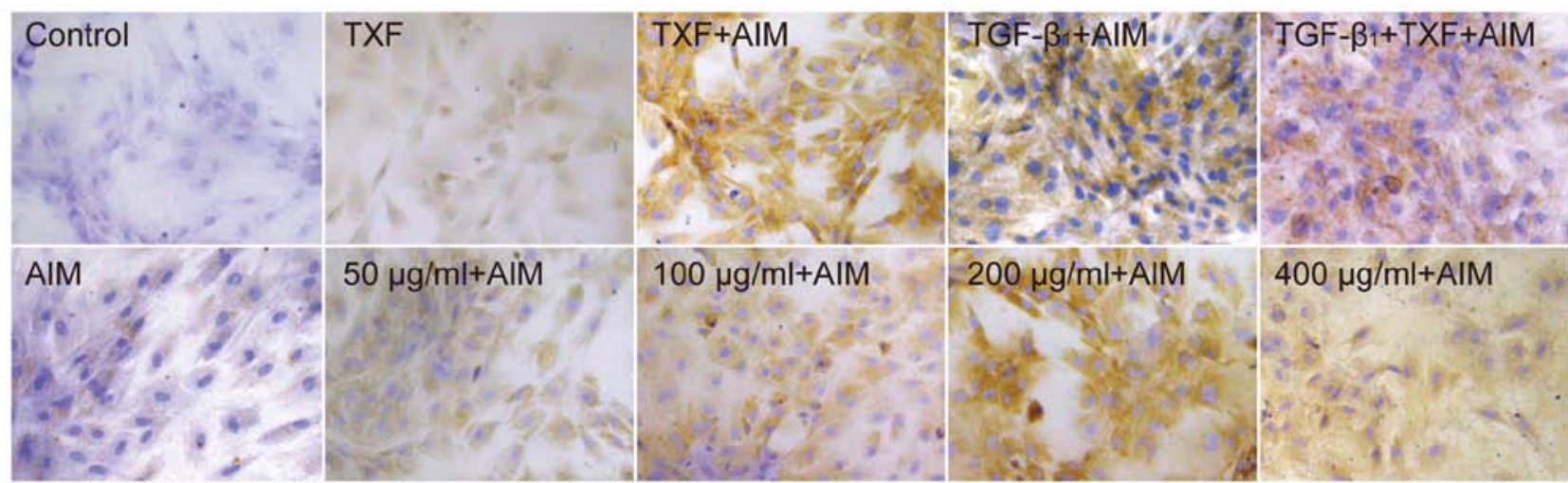

Figure 6. Immunohistochemistry detection of chondroblast-like cells. All the groups were treated once every 2 days for 2 weeks, and phosphate-buffered saline (PBS) was used to replace the collagen II as the negative control. Bone marrow stromal cells (BMSCs) were incubated in auxiliary-induced medium (AIM) and treated with 50, 100, 200 and $400 \mu \mathrm{g} / \mathrm{ml}$ of Tougu Xiaotong formula (TXF) for 2 weeks. Images were captured at a magnification, x100.
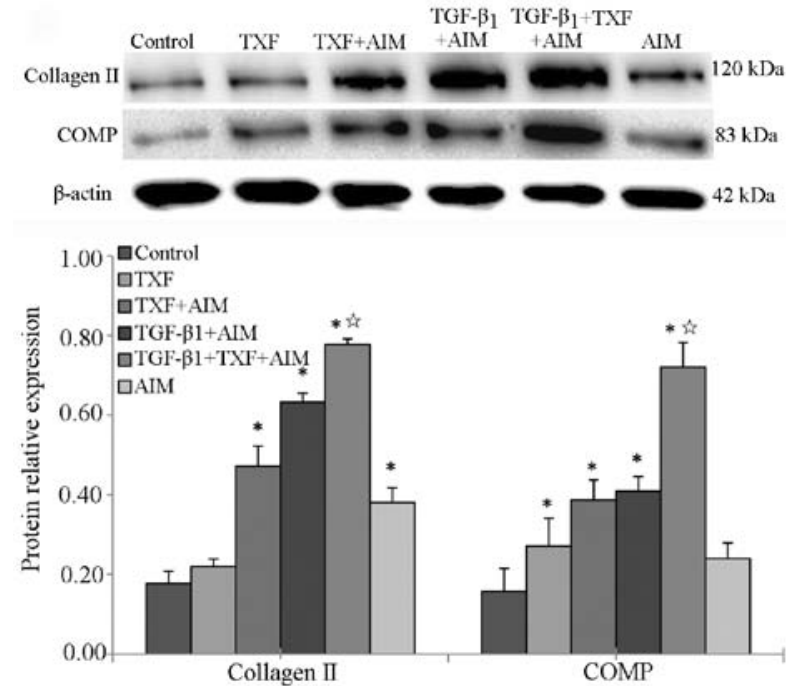

Figure 7. Tougu Xiaotong formula (TXF) increases the expression of collagen II and cartilage oligomeric matrix protein (COMP) in the chondrogenic differentiation of bone marrow stromal cells (BMSCs). The protein expression of collagen II and COMP were analyzed by western blotting, and $\beta$-actin was used as the internal control. The data shown are mean \pm standard deviation; ${ }^{*} \mathrm{P}<0.05$, vs. control group; ${ }^{\text {t }} \mathrm{P}<0.05$, vs. the transforming growth factor- $\beta 1$ (TGF- $\beta 1)+$ auxiliary induced medium (AIM) group.

the amount of collagen II positive cells in a dose-dependent manner, with the highest level of collagen II positive cells at $200 \mu \mathrm{g} / \mathrm{ml}$ TXF (Fig. 6). Taken together, the results indicated that TXF promoted chondrogenic differentiation of BMSCs in AIM, but independent application of TXF did not induce differentiation of BMSCs into chondrocytes.

TXF induces chondrogenic differentiation of BMSCs in association with TGF- $\beta 1$. COMP, the main composition of articular cartilage, plays an important role in maintaining the stability of joint structure (25). For chondrogenic differentiation of BMSCs, COMP is another important indicator. The protein expression of collagen II and COMP was analyzed by western blotting. Data from the western blot assay showed that the protein expression of collagen II and COMP in the AIM, $\mathrm{TXF}+\mathrm{AIM}, \mathrm{TGF}-\beta 1+\mathrm{AIM}$ and TGF- $\beta 1+\mathrm{TXF}+\mathrm{AIM}$ groups were significantly higher compared to the control

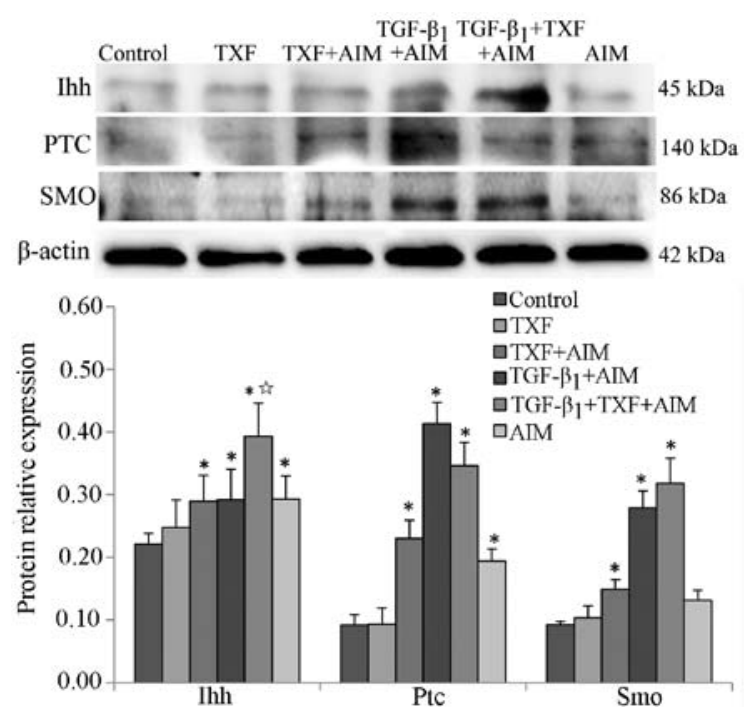

Figure 8. Tougu Xiaotong formula (TXF) upregulates the expression of Indian hedgehog (Ihh), Patched (Ptc) and Smoothened (Smo) in the chondrogenic differentiation of bone marrow stromal cells (BMSCs). The protein expression levels of Ihh, Ptc and Smo were determined by western blotting, $\beta$-actin was used as the internal control. The data shown are mean \pm standard deviation; ${ }^{*} \mathrm{P}<0.05$, vs. control group; ${ }^{\text {त }} \mathrm{P}<0.05$, vs. the transforming growth factor- $\beta 1$ (TGF- $\beta 1)+$ auxiliary induced medium (AIM) group.

group $(\mathrm{P}<0.05)$, and the highest levels of collagen II and COMP expression were in the TGF- $\beta 1+$ TXF + AIM group $(\mathrm{P}<0.05$, significant vs. TGF- $\beta 1+$ AIM group) (Fig. 7). Taken together, the results indicated that TXF induced chondrogenic differentiation of BMSCs in association with TGF- $\beta 1$.

Ih signaling pathway regulates the chondrogenic differentiation of BMSCs. A previous study showed that Ihh enhanced the expression of type II collagen in the process of human mesenchymal stem cells promoting meniscal regeneration (26). In the present study, the influence of TXF on the protein expression of the key regulators of Ihh signaling pathway was assessed in the BMSCs undergoing chondrogenic differentiation by western blotting. The protein expression levels of Ihh, Ptc and Smo in the TXF + AIM, TGF- $\beta 1+$ AIM, TGF- $\beta 1+$ TXF + AIM and AIM groups were significantly increased compared to the control group $(\mathrm{P}<0.05)$ (Fig. 8). In addition, the protein level of 
Ihh in the TGF- $\beta 1+$ TXF + AIM group was higher than that of the TGF- $\beta 1+$ AIM group $(\mathrm{P}<0.05)$. There was no significant difference of the protein levels of Smo and Ptc between the TGF- $\beta 1+\mathrm{TXF}+\mathrm{AIM}$ and TGF- $\beta 1+$ AIM groups $(\mathrm{P}>0.05)$. Taken together, the results indicated that differentiation of BMSCs into chondroblasts is associated with the Ihh pathway, and TXF-induced chondrogenic differentiation of BMSCs is associated with TGF- $\beta 1$ by activating the Ihh signaling pathway.

\section{Discussion}

Previously, cell-based cartilage tissue engineering provided a challenge for the treatment of cartilage injury (27), among which BMSCs are seed cells with the highest potential for articular cartilage repair in tissue engineering due to the extensive expansion capacity and multipotential differentiation. A number of studies demonstrated that certain effective components of Chinese herbs induced the differentiation of BMSCs into chondrocytes (7-9). In the present study, we hypothesized that TXF promoted chondrogenic differentiation of BMSCs. The data suggested that TXF induced chondrogenic differentiation of BMSCs in association with TGF- $\beta 1$ via activating the Ihh signaling pathway. In addition, TXF also promoted BMSCs proliferation by upregulating the expression of cyclin D1, CDK4 and CDK6.

To evaluate the side-effect of TXF on the BMSCs, the cell viability was determined by the MTT assay. The data exhibited that treatment with TXF promoted BMSCs viability in a doseand time-dependent, indicating that TXF was not cytotoxic to BMSCs. To further explore the mechanism, its effect was examined for the $G_{1}$ to $S$ phase transition in BMSCs via PI staining followed by FACS analysis. The results exhibited that the percentage of the proportion of BMSCs in the $G_{0} / G_{1}$ and $S$ phases was significantly reduced and increased, respectively, following TXF treatment, indicating that TXF promotes the proliferation by promoting BMSCs from the $\mathrm{G}_{1}$ to the $\mathrm{S}$ phase in vitro. In addition, cyclin D1 forms complexes with CDK4 or CDK6 that may regulate the $G_{1} / S$ transition, which is one of the two main checkpoints used by a cell to control the progression of the cell cycle, and subsequently promote cell proliferation $(22,23,28)$. Treatment with TXF enhanced the mRNA and protein expression of cyclin D1, CDK4 and CDK6 in BMSCs. Taken together, the results indicated that TXF promoted BMSCs proliferation by upregulating the expression of cyclin D1, CDK4 and CDK6.

Collagen II is synthesized and secreted into the cartilage ECM (29). To further study the effect of TXF on the chondrogenic differentiation of BMSCs, collagen II in the ECM was determined by IHC in the present study. The TXF + AIM group increased the amount of collagen II in positive cells in a dose-dependent manner, while the independent application of TXF did not induce BMSCs into chondroblast-like cells. The results showed that TXF promoted chondrogenic differentiation of BMSCs in the special culture conditions containing AIM. Additionally, the study analyzed the expression levels of collagen II and COMP by western blotting. In cartilage, COMP acts as a molecular bridge in maintaining the interstitial collagen II network (30). The TXF + TGF- $\beta 1+$ AIM group significantly enhanced the protein expression of collagen II and COMP compared to the TGF- $\beta 1+$ AIM group. TGF- $\beta 1$, which can promote BMSCs proliferation and differentiation, is one member of the TGF- $\beta$ superfamily (31). The results indicated that TXF-induced BMSC chondrogenic differentiation in association with TGF- $\beta 1$ in vitro.

Hedgehog mainly has three types of homologous proteins in mammals, known as Ihh, Sonic hedgehog and Desert hedgehog (32). Ihh was reported to play an essential role in regulating chondrocyte maturation, hypertrophy and differentiation $(11,33)$. The previous study also showed that Ihh protein has the ability to promote differentiation of chondrogenic precursor cells (34), and inactivation of its membrane receptor, Ptc, in the mouse limb has novel inhibitory effects of cell autonomously-activated hedgehog signaling on chondrogenesis (35). In the present study, the TXF + AIM, TGF- $\beta 1+$ AIM, TGF- $\beta 1+$ TXF + AIM and AIM groups upregulated the protein expression of Ihh, Ptc and Smo compared to the control group, and the protein level of Ihh in the TGF- $\beta 1+\mathrm{TXF}+\mathrm{AIM}$ group was higher than that of the TGF- $\beta 1$ group. The results demonstrated that the chondrogenic differentiation of BMSCs was associated with the Ihh signaling pathway, and TXF induced BMSC chondrogenic differentiation in association with TGF- $\beta 1$ by increasing the expression of Ihh, Ptc and Smo.

In conclusion, the present study demonstrates that TXF promotes the proliferation by upregulating the expression of cyclin D1, CDK4 and CDK6, and induces BMSC chondrogenic differentiation in association with TGF- $\beta 1$ via activating the Ihh signaling pathways, suggesting that TXF is a potential therapeutic agent for bone and joint disease by promoting chondrogenic differentiation of BMSCs. As a result of the lack of empirical evidence, future studies focusing on investigating the influence of TXF on the chondrogenic differentiation of BMSCs in vivo when combined with the cartilage tissue engineering are necessary.

\section{Acknowledgements}

The present study was supported by the National Natural Science Foundation of China (grant nos. 81373818 and 81102609), the Key Project of Fujian Provincial Department of Science and Technology (grant no. 2014Y0064), the Natural Science Foundation of Fujian Province (grant no. 2014J01357), the Developmental Fund of Chen Keji Integrative Medicine (CKJ2014001) and the Special Research Fund for Doctor Discipline in College (20123519110001).

\section{References}

1. Pittenger MF, Mackay AM, Beck SC, et al: Multilineage potential of adult human mesenchymal stem cells. Science 284 : $143-147,1999$.

2. Charbord P: Bone marrow mesenchymal stem cells: historical overview and concepts. Hum Gene Ther 21: 1045-1056, 2010

3. Pittenger MF and Martin BJ: Mesenchymal stem cells and their potential as cardiac therapeutics. Circ Res 95: 9-20, 2004.

4. Dominici M, Le Blanc K, Mueller I, et al: Minimal criteria for defining multipotent mesenchymal stromal cells. The International Society for Cellular Therapy position statement. Cytotherapy 8: 315-317, 2006.

5. Li WJ, Tuli R, Okafor C, et al: A three-dimensional nanofibrous scaffold for cartilage tissue engineering using human mesenchymal stem cells. Biomaterials 26: 599-609, 2005.

6. Ochi M, Uchio Y, Tobita M and Kuriwaka M: Current concepts in tissue engineering technique for repair of cartilage defect. Artif Organs 25: 172-179, 2001. 
7. Li X, Wei G, Wang X, et al: Targeting of the Sonic Hedgehog pathway by atractylenolides promotes chondrogenic differentiation of mesenchymal stem cells. Biol Pharm Bull 35: $1328-1335,2012$

8. Lin J, Xiu Z and Wu Z: Effect of pilose antler polypeptides on the apoptosis of rabbit marrow mesenchymal stem cells differentiated into chondrogenic phenotype in vitro. Zhongguo Xiu Fu Chong Jian Wai Ke Za Zhi 20: 427-430, 2006 (In Chinese).

9. Huh JE, Park YC, Seo BK, et al: Cartilage protective and chondrogenic capacity of WIN-34B, a new herbal agent, in the collagenase-induced osteoarthritis rabbit model and in progenitor cells from subchondral none. Evid Based Complement Alternat Med 2013: 527561, 2013.

10. Mak KK, Kronenberg HM, Chuang PT, Mackem S and Yang Y: Indian hedgehog signals independently of PTHrP to promote chondrocyte hypertrophy. Development 135: 1947-1956, 2008.

11. St-Jacques B, Hammerschmidt $M$ and McMahon AP: Indian hedgehog signaling regulates proliferation and differentiation of chondrocytes and is essential for bone formation. Genes Dev 13: 2072-2086, 1999.

12. Wu Q, Zhang Y and Chen Q: Indian hedgehog is an essential component of mechanotransduction complex to stimulate chondrocyte proliferation. J Biol Chem 276: 35290-35296, 2001.

13. Hooper JE and Scott MP: The Drosophila patched gene encodes a putative membrane protein required for segmental patterning. Cell 59: 751-765, 1989.

14. Nakano Y, Guerrero I, HidalgoA, Taylor A, Whittle JR and Ingham PW: A protein with several possible membranespanning domains encoded by the Drosophila segment polarity gene patched. Nature 341: 508-513, 1989.

15. Taipale J, Cooper M K, Maiti T and Beachy PA: Patched acts catalytically to suppress the activity of Smoothened. Nature 418 892-897, 2002

16. Li X, Lang W, Ye H, et al: Tougu Xiaotong capsule inhibits the tidemark replication and cartilage degradation of papain-induced osteoarthritis by the regulation of chondrocyte autophagy. Int J Mol Med 31: 1349-1356, 2013.

17. Li XH, Wu MX, Ye HZ, et al: Experimental study on the suppression of sodium nitroprussiate-induced chondrocyte apoptosis by Tougu Xiaotong Capsule-containing serum. Chin J Integr Med 17: 436-443, 2011.

18. Zhang M, Xie R, Hou W, et al: PTHrP prevents chondrocyte premature hypertrophy by inducing cyclin-D1-dependent Runx2 and Runx3 phosphorylation, ubiquitylation and proteasomal degradation. J Cell Sci 122: 1382-1389, 2009.

19. Hwang SG, Song SM, Kim JR, Park CS, Song WK and Chun JS Regulation of type II collagen expression by cyclin-dependent kinase 6, cyclin D1, and p21 in articular chondrocytes. IUBMB Life 59: 90-98, 2007.

20. Neuhuber B, Swanger SA, Howard L, Howard L, Mackay A and Fischer I: Effects of plating density and culture time on bone marrow stromal cell characteristics. Exp Hematol 36: 1176-1185, 2008
21. Chen ZZ, Van Bockstaele DR, Buyssens N, et al: Stromal populations and fibrosis in human long-term bone marrow cultures. Leukemia 5: 772-781, 1991.

22. Nurse P: Ordering S phase and M phase in the cell cycle. Cell 79: 547-550, 1994.

23. Shen R, Wang X, Drissi H, Liu F, O'Keefe RJ and Chen D: Cyclin D1-cdk4 induce runx2 ubiquitination and degradation. J Biol Chem 281: 16347-16353, 2006.

24. Pufe T, Petersen W, Fändrich F, et al: Programmable cells of monocytic origin (PCMO): a source of peripheral blood stem cells that generate collagen type II-producing chondrocytes. J Orthop Res 26: 304-313, 2008.

25. Oldberg A, Antonsson P, Lindblom K and Heinegård D: COMP (cartilage oligomeric matrix protein) is structurally related to the thrombospondins. J Biol Chem 267: 22346-22350, 1992.

26. Horie M, Choi H, Lee RH, et al: Intra-articular injection of human mesenchymal stem cells (MSCs) promote rat meniscal regeneration by being activated to express Indian hedgehog that enhances expression of type II collagen. Osteoarthritis Cartilage 20: 1197-1207, 2012.

27. Hettrich CM, Crawford D and Rodeo SA: Cartilage repair: third-generation cell-based technologies - basic science, surgical techniques, clinical outcomes. Sports Med Arthrosc 16: 230-235, 2008.

28. Beier F: Cell-cycle control and the cartilage growth plate. J Cell Physiol 202: 1-8, 2005.

29. Ito H, Rucker E, Steplewski A, et al: Guilty by association: some collagen II mutants alter the formation of ECM as a result of atypical interaction with fibronectin. J Mol Biol 352: 382-395, 2005.

30. Agarwal P, Schulz JN, Blumbach K, et al: Enhanced deposition of cartilage oligomeric matrix protein is a common feature in fibrotic skin pathologies. Matrix Biol 32: 325-331, 2013

31. Miura Y, Parvizi J, Fitzsimmons JS and O'Driscoll SW: Brief exposure to high-dose transforming growth factor-beta1 enhances periosteal chondrogenesis in vitro: a preliminary report. J Bone Joint Surg Am 84-A: 793-799, 2002.

32. McMahon AP, Ingham PW and Tabin CJ: Developmental roles and clinical significance of hedgehog signaling. Curr Top Dev Biol 53: 1-114, 2003

33. Choi SW, Jeong DU, Kim JA, et al: Indian Hedgehog signalling triggers $\mathrm{Nkx} 3.2$ protein degradation during chondrocyte maturation. Biochem J 443: 789-798, 2012.

34. Enomoto-Iwamoto M, Nakamura T, Aikawa T, et al: Hedgehog proteins stimulate chondrogenic cell differentiation and cartilage formation. J Bone Miner Res 15: 1659-1668, 2000

35. Bruce SJ, Butterfield NC, Metzis V, Town L, McGlinn E and Wicking C: Inactivation of Patched 1 in the mouse limb has novel inhibitory effects on the chondrogenic program. J Biol Chem 285: 27967-27981, 2010. 